\title{
Estimation de l'épuisement des ressources hospitalières attribuable à la COVID-19 en Ontario, au Canada
}

\author{
Kali Barrett MD, Yasin A. Khan MD, Stephen Mac MBiotech, Raphael Ximenes PhD, David M.J. Naimark MD MSc, \\ Beate Sander PhD
}

Citation : CMAJ 2020 June 15;192:E640-6. doi: 10.1503/cmaj.200715-f; diffusion hâtive le 14 mai 2020

Pour des articles connexes, consulter le www.cmaj.ca/lookup/doi/10.1503/cmaj.200457 et le www.cmaj.ca/lookup/doi/10.1503/cmaj.200476

Voir la version anglaise de l'article ici : www.cmaj.ca/lookup/doi/10.1503/cmaj.200715

\section{RÉSUMÉ}

CONTEXTE : La propagation à l'échelle planétaire de la maladie à coronavirus 2019 (COVID-2019) se poursuit dans plusieurs pays, mettant à rude épreuve les systèmes de santé. Cette étude avait pour but de prédire les répercussions de la pandémie sur les issues des patients et l'utilisation des ressources hospitalières en Ontario (Canada).

MÉTHODES : Nous avons conçu un modèle de simulation axé sur les personnes illustrant le flux de patients atteints de la COVID-19 dans les hôpitaux ontariens. Nous avons simulé diverses combinaisons de trajectoires épidémiques et de capacités de soins hospitaliers. Les paramètres à l'étude étaient le nombre de patients devant être admis au service d'hospitalisation ou à l'unité des soins intensifs (USI) - avec ou sans respirateur mécanique -, le nombre de jours jusqu'à l'épuisement des ressources, le nombre de patients en attente de ressources et le nombre de décès.

RÉSULTATS : Nous avons constaté que la mise en place rapide de mesures de santé publique efficaces éviterait l'épuisement des ressources hospitalières. Les simulations dans lesquelles les mesures d'éloignement sanitaire étaient inefficaces ou adoptées tardivement ont montré que l'épuisement des ressources prendrait de 14 à 26 jours et qu'il y aurait, dans le pire des cas, 13321 décès de personnes en attente de ressources. Cet épuisement pourrait être évité ou retardé par la mise en place de mesures rigoureuses visant à améliorer la capacité des hôpitaux en matière de soins intensifs, de respirateurs mécaniques et de soins hospitaliers.

INTERPRÉTATION : Sans l'adoption de mesures d'éloignement sanitaire rigoureuses, le système de santé ontarien n'aurait pas eu les ressources nécessaires pour prendre en charge le nombre attendu de patients atteints de la COVID-19, même en cas d'augmentation rapide de sa capacité hospitalière. Les pénuries auraient fait augmenter le taux de mortalité. En ralentissant la transmission de la maladie par la mise en place de mesures de santé publique et l'augmentation de la capacité des hôpitaux, l'Ontario a probablement évité que ces derniers subissent une pression catastrophique.
$\mathbf{L}$

e 11 mars 2020, l'Organisation mondiale de la Santé (OMS) a déclaré que la propagation mondiale du coronavirus du syndrome respiratoire aigu sévère 2 (SRAS-CoV-2) et de la maladie qu'il provoque, la maladie à coronavirus 2019 (COVID-19), était une pandémie ${ }^{1,2}$. Le 26 avril 2020, 2,9 millions de cas confirmés et près de 200000 décès avaient été recensés dans le monde ${ }^{3,4}$. Fait inquiétant : les 100000 premiers cas sont apparus en 3 mois, et les 100000 cas suivants, en 12 jours seulement ${ }^{5}$. Afin de réduire la transmission de la maladie, une grande partie de la population mondiale a déployé des efforts pour réduire au minimum les interactions sociales (éloignement sanitaire), et certains pays ont procédé de façon dynamique au dépistage et à la recherche de contacts ${ }^{6}$. Sans l'adoption de telles mesures préventives, les besoins de soins intensifs auraient été plus grands que les ressources accessibles ${ }^{7,8}$.

Il est important d'anticiper la propagation de la COVID-19 et d'évaluer les interventions visant à atténuer cette propagation pour que les systèmes de santé soient bien préparés à affronter la pandémie. Plusieurs modèles de transmission ont indiqué que l'absence d'éloignement sanitaire rigoureux aurait mené à l'épuisement des ressources disponibles ${ }^{9,10}$. Bien que ces modèles donnent un aperçu de l'ampleur de la propagation de la COVID-19 selon divers scénarios et permettent une estimation générale de l'utilisation des ressources, ils ne s'adaptent pas de façon dynamique aux fluctuations des ressources (ils adoptent 
plutôt une approche linéaire); ils ne tiennent donc pas compte des changements dans les ressources qui découlent des décès, des guérisons, du temps d'attente et de l'établissement de critères de priorité chez les personnes en attente.

En ce qui a trait à la COVID-19, des données montrent qu'une capacité hospitalière moindre est associée à un taux de mortalité accru $^{11}$. Au Canada, il est particulièrement pertinent de connaître les répercussions de la COVID-19 sur les ressources hospitalières: en 2018, on y comptait 2,5 lits d'hôpital pour 1000 habitants, comparativement à 4,34 en Chine, à 3,84 en Australie, à 3,18 en Italie et à 2,54 au Royaume-Uni ${ }^{12}$.

Nous avions pour objectif d'anticiper les répercussions de la COVID-19 sur le système hospitalier et le taux de mortalité en Ontario (Canada), en fonction de divers scénarios d'infection et de capacité hospitalière, pour montrer quelles auraient été les conséquences systémiques de la maladie selon des scénarios pessimiste et optimiste, et savoir à quoi s'attendre en réalité dans cette province.

\section{Méthodes}

\section{Conception de l'étude et sources de données}

Nous avons élaboré un modèle de transition de l'état de santé en temps discret axé sur les personnes afin de prévoir l'utilisation des ressources hospitalières auprès des patients atteints de la COVID-19. Les principaux paramètres à l'étude étaient le nombre de patients devant être admis en soins hospitaliers ou intensifs - sans égard au besoin de respirateur mécanique -, le nombre de jours jusqu'à l'épuisement des ressources, le nombre de patients en attente de l'une ou l'autre de ces ressources par jour, et le nombre de personnes hospitalisées décédées de la COVID19 , stratifié en fonction de l'accès ou du non-accès aux ressources nécessaires. Les résultats ont été comptabilisés sur une période de 60 jours (2 mois), et la durée du cycle du modèle (intervalle de temps) a été exprimée en jours.

Pour la simulation, nous avons utilisé une population dynamique d'adultes (18 ans et plus) atteints de la forme symptomatique de la COVID-19 se présentant au service des urgences. Certains étaient renvoyés à la maison, où ils devaient s'isoler, et d'autres étaient hospitalisés (figure 1). Les patients hospitalisés étaient admis dans un service de soins hospitaliers ou à l'unité de soins intensifs (USI), selon la gravité de leur état. Certains patients de l'USI avaient besoin d'un respirateur mécanique. Dans ce modèle, les patients pouvaient maintenir un état stable, se rétablir ou décéder.

Advenant le cas où une ressource (p. ex., lit d'hôpital, lit de soins intensifs, respirateur mécanique) était non disponible, nous avons supposé que les patients étaient gardés là où ils se trouvaient (état des ressources) en attendant que la ressource requise se libère. Par exemple, un patient qui attendait qu'un lit de I'USI se libère pour y être admis restait au service des urgences entre-temps, où il n'avait accès à aucune ressource de

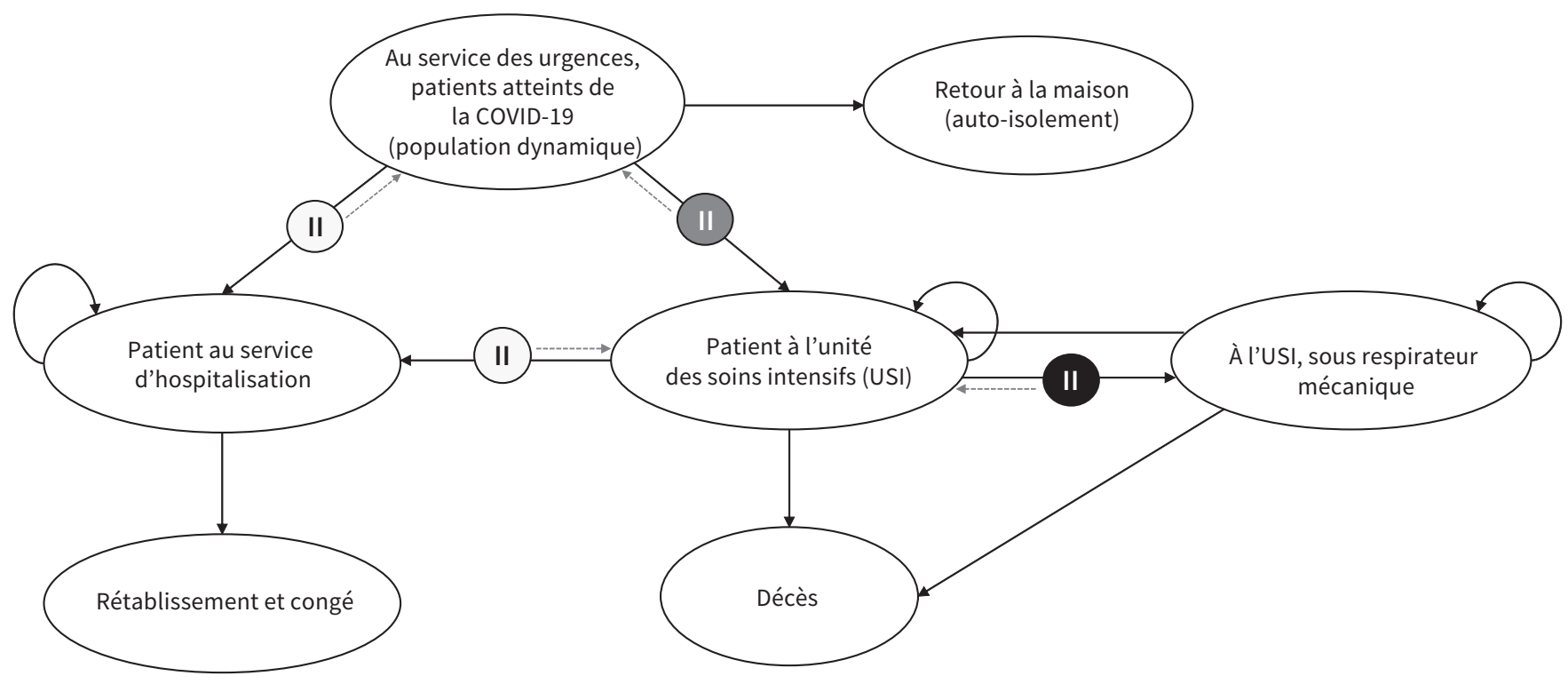

(II) Ce symbole représente une contrainte en matière de lits d'hôpital. Si aucun lit n'est disponible, le patient reste là où il se trouvait à l'étape précédente, comme l'indique la flèche pointillée.

(I) Ce symbole représente une contrainte en matière de lits en USI. Si aucun lit n'est disponible, le patient reste là où il se trouvait à l'étape précédente, comme l'indique la flèche pointillée.

II Ce symbole représente une contrainte en matière de respirateurs mécaniques. Si aucun respirateur n'est disponible, le patient reste là où il se trouvait à l'étape précédente, comme l'indique la flèche pointillée; on présume que le patient décédera au cours de la journée.

Figure 1: Schéma du modèle. COVID-19 = maladie à coronavirus 2019; USI = unité de soins intensifs. 
soins intensifs. Un respirateur ou un lit de soins intensifs se libérait après la guérison ou le décès d'un patient, tandis qu'un lit d'hôpital se libérait une fois le patient rétabli. La priorité d'accès aux lits d'hôpital était déterminée en fonction de l'endroit où se trouvaient les patients (les patients de l'USI passaient avant ceux du service des urgences). Pour les autres ressources, la priorité était donnée aux patients ayant attendu le plus longtemps depuis leur admission. Toutes les modélisations et les analyses ont été réalisées avec TreeAge Pro 2020 (TreeAge Software).

\section{Évolution de la COVID-19}

Les données ont été extraites de la littérature scientifique grandissante sur la COVID-19 et complétées par des experts (tableau 1).

Nous avons présumé que la probabilité qu'un adulte atteint de la forme symptomatique de la COVID-19 soit admis dans un hôpital ontarien correspondait au taux brut d'hospitalisation estimé au Canada, soit à $18 \%{ }^{13}$. Comme nous ne disposions d'aucune donnée propre à l'Ontario, nous avons estimé, par calage du taux d'occupation observé des USI, que le taux d'admission en soins intensifs en cas d'hospitalisation était de $48 \%$. Nous avons mené une recherche par grille rudimentaire, les techniques de calage plus systématiques, comme le recuit simulé et l'algorithme de Nelder-Mead, supposant l'existence de données de grande qualité, ce que nous n'avions pas à ce moment. L'estimation du taux d'Ontariens admis en soins intensifs ayant besoin d'un respirateur mécanique était de $78 \%$, selon les données de Services ontariens des soins aux malades en phase critique au 13 avril $2020^{14}$. Nous avons présumé que seuls les patients en soins intensifs couraient un risque de décès durant leur hospitalisation ${ }^{11}$, et avons appliqué le même risque de décès aux patients qui avaient besoin de soins intensifs sans respirateur mécanique, mais qui n'avaient pas accès à un lit de l'USI en raison de contraintes en matière de ressources. Nous avons également supposé que les patients qui avaient besoin d'un respirateur mécanique, mais qui ne pouvaient pas l'obtenir, décédaient le jour même. La probabilité de décès à I'USI (sans égard au besoin de respirateur mécanique) était basée sur la proportion de décès $(35 \%)$ chez les patients présentant une forme modérée du syndrome de détresse respiratoire aiguë (SDRA), dont la durée moyenne de séjour à l'USI était de 11 jours (et la durée moyenne d'hospitalisation, de 17 jours), proportion rapportée dans une étude menée sur plusieurs pays ${ }^{15}$. Si les estimations de la durée d'hospitalisation et du taux de mortalité des patients atteints d'une forme modérée du SDRA ont été utilisées, c'est parce que le tableau clinique de ces patients s'apparente, selon les experts, à celui des patients hospitalisés à l'USI en raison de la COVID-19.

\section{Répercussions de la COVID-19 sur la disponibilité des ressources hospitalières}

En l'absence de traitements efficaces contre la COVID-19, il est possible de maximiser les issues de santé souhaitées en réduisant le nombre de cas par l'adoption de mesures de santé publique et en augmentant la capacité du système (p. ex., en libérant les ressources existantes ou en ajoutant des ressources). Nous avons étudié 9 scénarios tenant compte de la propagation de la COVID-19 et de la capacité du système, basés sur 3 possibles trajectoires épidémiques et 3 scénarios de disponibilité des ressources.

Tableau 1 : Principales variables de la modélisation

\section{Variable}

Nombre de cas de COVID-19 déclarés en Ontario, par jour et par scénario

Probabilité qu'une hospitalisation soit nécessaire

Probabilité que des soins intensifs soient nécessaires en cas d'hospitalisation

Probabilité qu'un patient de l'USI ait besoin d'un respirateur mécanique

Probabilité que l'état d'un patient en soins hospitaliers se détériore et que des soins intensifs soient nécessaires

Durée de séjour en soins hospitaliers (pas d'admission à l'USI)

Durée de séjour en soins intensifs (avec ou sans respirateur mécanique)

Durée de séjour en soins hospitaliers après un séjour à l'USI

Probabilité de décès en soins hospitaliers

Probabilité de décès en soins intensifs

Probabilité de décès d'un patient sous respirateur mécanique

Probabilité de décès d'un patient en attente de respirateur mécanique
Valeur de

référence

Voir la figure 2

0,18

0,48

0,78

0

17 jours

11 jours

6 jours

0

0,35

0,35

1.0

\section{Source}

Estimation selon la méthode décrite dans le texte.

Estimation de l'Agence de la santé publique du Canada ${ }^{13}$

Calage selon l'estimation de l'Agence de la santé publique du Canada ${ }^{13}$

Estimation de SOSMPC au 13 avril $2020^{14}$

Hypothèse

Bellani et coll., $2016^{15}$

Bellani et coll., $2016^{15}$

Bellani et coll., $2016^{15}$

Hypothèse reposant sur Wu and McGoogan 202011

Bellani et coll., $2016^{15}$

Bellani et coll., $2016^{15}$

Hypothèse

Note : COVID-19 = maladie à coronavirus 2019, SOSMPC = Services ontariens des soins aux malades en phase critique, USI = unité de soins intensifs 


\section{Trajectoires épidémiques}

Nous avons modélisé 3 possibles trajectoires épidémiques pour l'Ontario à partir du 5 mars 2020, alors que la province comptait au total 102 cas de COVID-19, définis comme des cas confirmés déclarés (soit un résultat positif au dépistage du SRAS-CoV-2).

\section{Scénario optimiste}

Nous nous sommes servi des données quotidiennes d'incidence déclarées par la Corée du Sud pendant 60 jours (jusqu'au 23 avril 2020), avec un point de départ à 104 cas cumulatifs correspondant au point de départ de l'Ontario.

\section{Scénario attendu}

Partant de 102 cas cumulatifs au 5 mars 2020, nous nous sommes servis de données d'observation tirées du système intégré d'information sur la santé publique de l'Ontario jusqu'au 14 avril pour tenir compte des retards dans la déclaration des cas confirmés, puis avons fait diminuer de $5 \%$ (taux de contraction) le nombre de nouveaux cas par jour jusqu'au $60^{\mathrm{e}}$ jour. Un pic a été observé le 13 avril. Le taux de contraction représentait l'effet des mesures de santé publique adoptées à la mi-mars.

\section{Scénario pessimiste}

Nous nous sommes servi des données quotidiennes d'incidence déclarées par l'Italie pendant 60 jours (jusqu'au 23 avril 2020) ${ }^{3}$, en partant de 155 cas cumulatifs, ce qui correspondait le mieux au nombre de cas en Ontario à la date de départ.

L'évolution du nombre de cas est présentée à la figure 2. Le nombre de cas attendus chaque jour se trouve à l'annexe 1 (accessible en anglais au www.cmaj.ca/lookup/suppl/doi:10.1503/cmaj.200715/-/DC1).

\section{Ressources hospitalières}

Nous avons tenu compte du nombre de lits d'hôpital et du nombre de lits de soins intensifs, avec et sans respirateur. Pour qu'un patient puisse faire l'objet d'une ventilation mécanique, un respirateur et un lit de soins intensifs devaient tous deux être disponibles. Nous avons présumé que la dotation en personnel était adéquate dans toutes les unités. Notre modélisation repose sur 3 scénarios de disponibilité des ressources: scénario de référence, avec capacité d'appoint et augmentation massive.

\section{Scénario de référence}

Le nombre de lits avec respirateur disponibles au début de la simulation représente le nombre de respirateurs existant en Ontario. Nous avons présumé, en nous fondant sur des avis d'experts, que $25 \%$ du nombre total de lits avec respirateur (328/1311) et de lits de soins intensifs sans respirateur (186/742) et $20 \%$ du nombre de lits d'hôpital (4000/20000) seraient disponibles pour les patients atteints de la COVID-19, et qu'aucun lit ou respirateur ne serait ajouté.

\section{Scénario avec capacité d'appoint}

Nous avons ajouté au scénario de référence une capacité d'appoint de 502 lits avec respirateur, de 351 lits de soins intensifs sans respirateur et de 1351 lits d'hôpital (total : 830 lits avec respirateur, 537 lits de soins intensifs sans respirateur et 5351 lits d'hôpital). Ce scénario maximise les ressources disponibles par la réduction des activités cliniques et l'activation des protocoles de capacité d'appoint (Erik Hellsten, Santé Ontario, Toronto : communication personnelle, 2020).

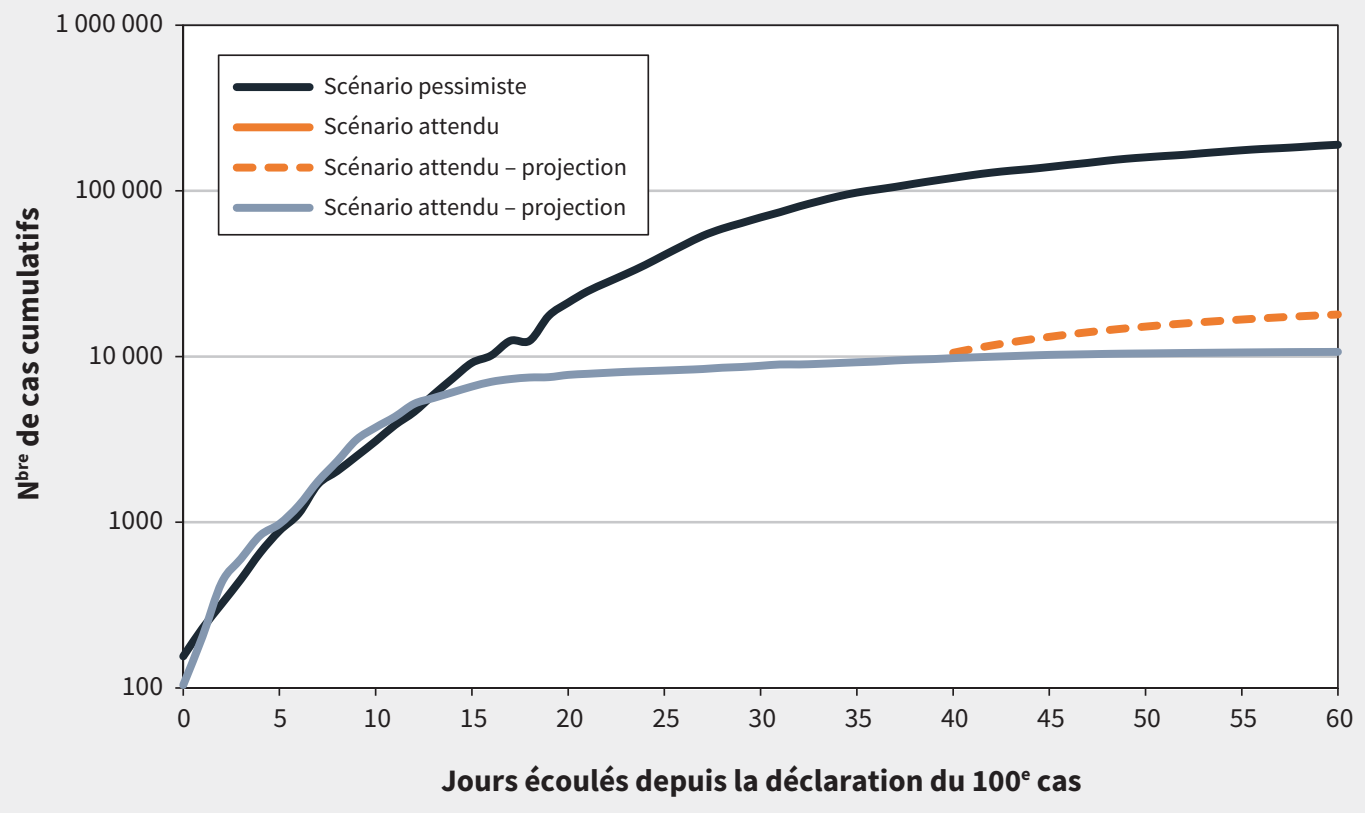

Figure 2: Nombre cumulatif de cas de maladie à coronavirus 2019 (COVID-19) en Ontario. 


\section{Scénario d'augmentation massive}

Dans ce scénario, la capacité du système de santé a été considérablement accrue en augmentant l'espace hospitalier par des moyens non conventionnels. Par conséquent, 2000 lits de soins intensifs avec respirateur, 1000 lits de soins intensifs sans respirateur et 10000 lits d'hôpital seraient disponibles pour les patients atteints de la COVID-19.

\section{Résultats}

\section{Trajectoires épidémiques}

Selon la trajectoire épidémique la plus optimiste, et en supposant que les ressources disponibles correspondent au scénario de référence, on anticipe un total de 10579 cas de COVID-19 en 60 jours, dont 1904 nécessiteraient une hospitalisation (tableau 2). Les ressources que sont les lits de soins intensifs, les respirateurs et les lits d'hôpital seraient particulièrement sollicitées du jour 10 au jour 20, mais ne seraient jamais épuisées (annexe 1). Selon ce scénario, 353 patients seraient décédés de la COVID 19, malgré un accès approprié aux soins et aux ressources hospitalières.

Selon la trajectoire épidémique attendue, toujours en supposant que les ressources disponibles correspondent au scénario de référence, on anticipe un total de 18047 cas de COVID-19 en 60 jours, dont 3248 nécessiteraient une hospitalisation. D'après les données d'observation de l'Ontario, où des mesures d'éloignement sanitaire ont été mises en place le 15 mars, les ressources en matière de lits de soins intensifs, de respirateurs et de lits d'hôpital ne seraient pas épuisées (figure 3). Selon ce scénario, 528 patients seraient décédés de la COVID-19, malgré un accès approprié aux soins et aux ressources hospitalières.

Après calage, le taux d'occupation des lits de soins intensifs et d'hôpital projeté au moyen de ce scénario était plus optimiste que celui indiqué par les données d'observation provinciales (figure 4).
Quant à la trajectoire épidémique la plus pessimiste, si on suppose toujours que les ressources disponibles correspondent au scénario de référence, elle mènerait à un total anticipé de 192839 cas de COVID-19 en 60 jours, dont 34711 nécessiteraient une hospitalisation. L'épuisement des ressources en matière de lits de soins intensifs et de respirateurs surviendrait après 19 jours et 14 jours, respectivement. Les unités de soins auraient été incapables d'accepter de nouveaux patients après 26 jours (annexe 1 ). Étant donné le grand nombre quotidien de cas de COVID-19 et l'épuisement rapide des ressources mis en évidence dans ce scénario, 13321 patients en attente de soins appropriés et 1060 patients ayant reçu les soins nécessaires seraient décédés.

\section{Scénarios d'augmentation des ressources}

La trajectoire épidémique la plus pessimiste était le seul scénario dans lequel les ressources de référence auraient été insuffisantes. Avec l'augmentation des ressources, l'augmentation du nombre de lits de soins intensifs et de respirateurs retarderait l'épuisement des ressources, mais ne l'éliminerait pas (annexe 1). Par exemple, selon le scénario avec capacité d'appoint, l'épuisement des ressources en matière de lits de soins intensifs et de respirateurs surviendrait après 25 jours et 19 jours, respectivement, plutôt que 17 jours et 14 jours, respectivement.

\section{Mortalité}

En supposant que les ressources disponibles correspondent au scénario de référence, la pire trajectoire épidémique entraînerait le décès de 13321 patients en attente de ressources. Cependant, l'augmentation du nombre de ressources réduirait ce nombre à 8605 dans le scénario avec capacité d'appoint et à 1876 dans le scénario d'augmentation massive en matière de ressources (tableau 2).

Tableau 2 : Relation entre la trajectoire épidémique, la disponibilité des ressources et la mortalité

\begin{tabular}{|c|c|c|c|c|c|}
\hline \multirow[b]{2}{*}{ Scénario de ressources } & \multicolumn{3}{|c|}{$\begin{array}{c}N^{\text {bre }} \text { de jours jusqu'à l'épuisement } \\
\text { des ressources }\end{array}$} & \multicolumn{2}{|c|}{$\mathrm{N}^{\mathrm{bre}}$ de décès } \\
\hline & $\begin{array}{l}\text { Lits de soins } \\
\text { intensifs }\end{array}$ & Respirateurs & $\begin{array}{l}\text { Lit } \\
\text { d'hôpital }\end{array}$ & $\begin{array}{l}\text { En attente de } \\
\text { ressources }\end{array}$ & $\begin{array}{c}\text { En ayant les } \\
\text { ressources } \\
\text { nécessaires }\end{array}$ \\
\hline \multicolumn{6}{|l|}{ Scénario de référence } \\
\hline Scénario optimiste & Jamais & Jamais & Jamais & 0 & 353 \\
\hline Scénario attendu & Jamais & Jamais & Jamais & 0 & 528 \\
\hline Scénario pessimiste & 19 & 14 & 26 & 13321 & 1060 \\
\hline \multicolumn{6}{|c|}{ Scénario avec capacité d'appoint } \\
\hline Scénario optimiste & Jamais & Jamais & Jamais & 0 & 353 \\
\hline Scénario attendu & Jamais & Jamais & Jamais & 0 & 528 \\
\hline Scénario pessimiste & 25 & 19 & 28 & 8605 & 2608 \\
\hline \multicolumn{6}{|c|}{ Scénario d'augmentation massive } \\
\hline Scénario optimiste & Jamais & Jamais & Jamais & 0 & 353 \\
\hline Scénario attendu & Jamais & Jamais & Jamais & 0 & 528 \\
\hline Scénario pessimiste & 34 & 27 & Jamais & 1876 & 4966 \\
\hline
\end{tabular}




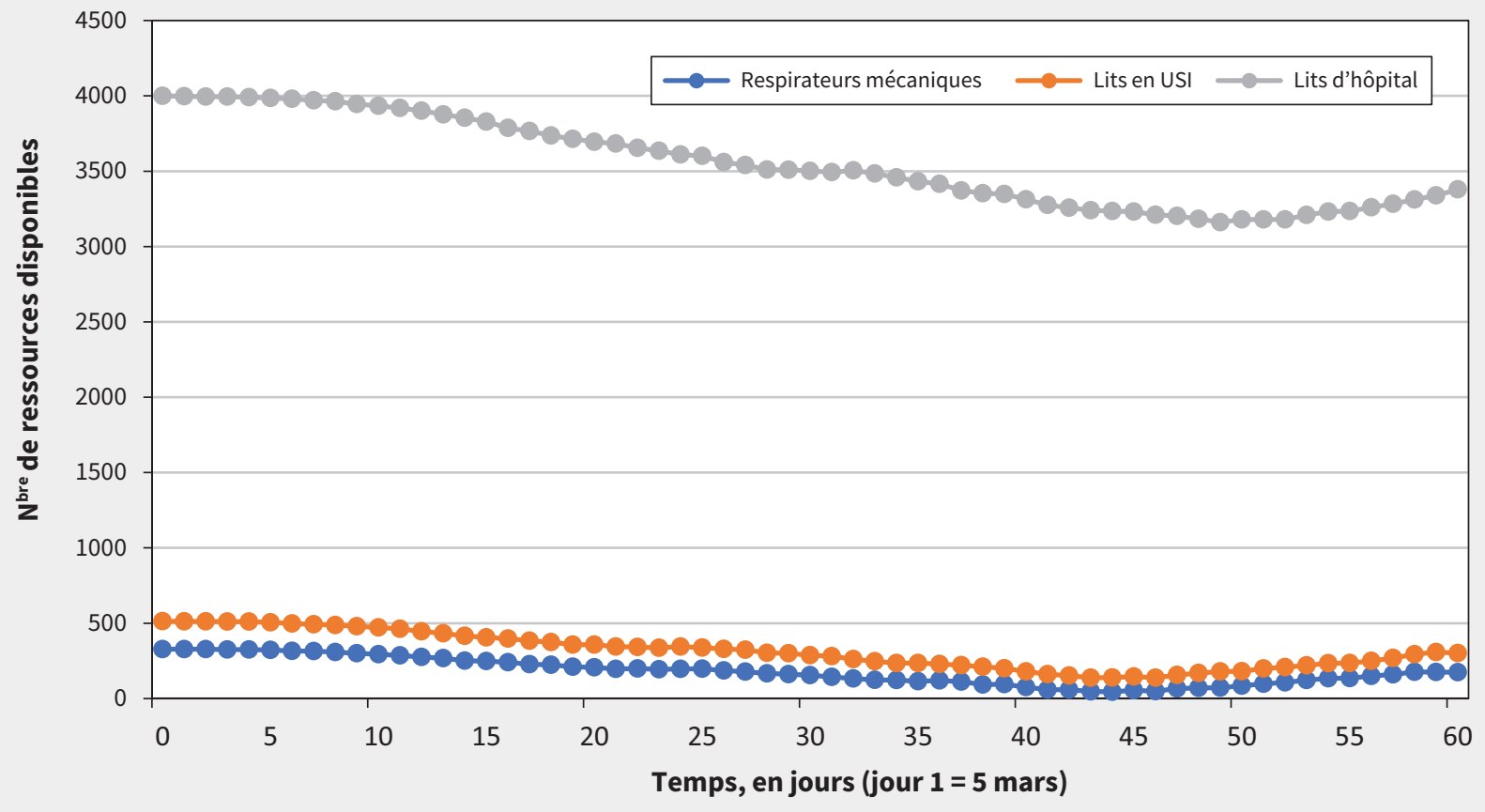

Figure 3 : Épuisement des ressources si la trajectoire épidémique est celle attendue et si les ressources disponibles correspondent au scénario de référence. USI = unité de soins intensifs.

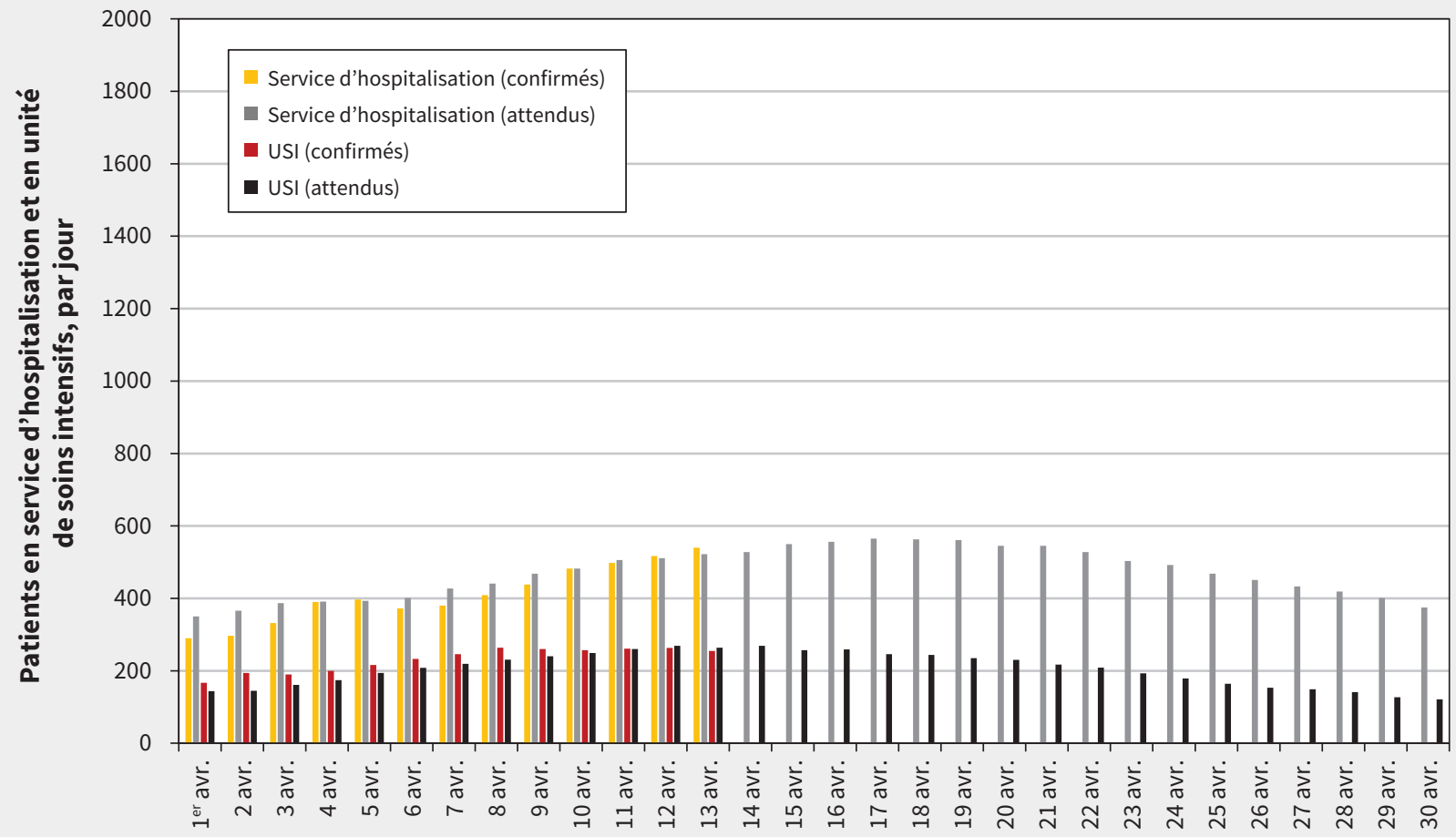

Date

Figure 4 : Occupation attendue et confirmée des services d'hospitalisation et des unités de soins intensifs par les patients atteints de la maladie à coronavirus 2019 (COVID-19) en Ontario. 


\section{Interprétation}

Nos résultats montrent que des mesures de santé publique combinées à l'accroissement de la capacité du système de santé en matière de ressources hospitalières réduiraient ou retarderaient considérablement l'effondrement du système et l'épuisement des ressources.

Au 13 avril 2020, le taux d'occupation des lits de soins intensifs par les patients atteints de la COVID-19 anticipé par la trajectoire épidémique attendue de notre modèle correspondait bien à celui observé en Ontario, qui est resté stable pendant 3 semaines (du 6 au 27 avril), et semble confirmer l'efficacité des interventions de santé publique. Si toutefois l'Ontario n'avait pas adopté ces interventions et avait suivi les traces de l'Italie, la capacité des soins intensifs aurait été dépassée de façon rapide et catastrophique, même avec l'augmentation massive des ressources, et 13321 patients seraient décédés en raison des pénuries. Bien que l'Ontario ait évité l'effondrement de son système hospitalier, notre modèle montre qu'un allègement de la pression exercée sur les ressources et une diminution du nombre de décès auraient été possibles dans le meilleur des cas, par exemple dans un scénario ressemblant à ce qui s'est passé en Corée du Sud.

Deux autres études de modélisation se sont penchées sur la COVID-19 en Ontario et au Canada ${ }^{10,16}$. Dans les 2 cas, des modèles de transmission ont été élaborés pour déterminer les trajectoires épidémiques potentielles de la maladie et prévoir le taux d'occupation des lits de soins intensifs, mais on s'est servi de données relativement agrégées pour estimer la disponibilité des ressources ${ }^{10,16}$. Notre modèle, quant à lui, tient compte des contraintes en matière de ressources ainsi que d'une augmentation de la capacité en soins intensifs, ce qui nous permet de mettre en évidence l'augmentation du nombre de décès associée à l'épuisement des ressources.

Notre étude a plusieurs forces. Outre la modélisation de diverses trajectoires pandémiques, elle comprend également des scénarios d'augmentation de la capacité hospitalière, présentant et quantifiant explicitement les effets de ces augmentations. Nos estimations diffèrent de celles des autres études parce que la mortalité des patients y est stratifiée en fonction de la réception ou de la nonréception de soins appropriés. Notre étude modélise également les transitions des patients au sein de l'hôpital pour estimer l'utilisation et la disponibilité des ressources à chaque niveau de soins, ce qui nous a permis de prédire l'effet de l'épuisement des ressources sur la mortalité. Sa validité est renforcée par l'intégration de données d'observation venant de pays où des mesures énergiques de santé publique ont été mises en place rapidement, ainsi que d'autres pays où de telles mesures manquaient à l'appel. Enfin, nous avons obtenu des estimations à jour en ce qui concerne les ressources de santé locales et avons estimé l'augmentation de ces ressources en cas de capacité accrue à l'aide de données tirées de propositions faites par les gouvernements et les systèmes de santé.

\section{Limites de l'étude}

Notre étude présente toutefois certaines faiblesses. Notre modèle repose sur la prédiction du nombre de cas de COVID-19 en fonction des données disponibles ainsi que des projections et des hypothèses relatives à l'efficacité de l'éloignement sanitaire. L'hypothèse selon laquelle les patients admis dans un service d'hospitalisation ne mourraient pas de la COVID-19, hypothèse fondée sur la littérature scientifique actuelle, peut nous avoir amenés à sous-estimer le nombre de décès à l'hôpital. Nous avons présumé, pour refléter les données disponibles au moment de l'étude, qu'il était impossible que l'état des patients admis dans un service d'hospitalisation se dégrade, et avons posé l'hypothèse simpliste selon laquelle les patients gravement malades seraient repérés rapidement au service des urgences et admis directement à l'USI. Nous avons également supposé que le service des urgences disposerait de ressources illimitées et avons omis de tenir compte des limites de capacité. Par ailleurs, au moment de la modélisation, aucune donnée sur la durée d'hospitalisation des patients atteints de la COVID-19 en soins hospitaliers ou intensifs n'était disponible, et les connaissances quant aux répercussions de l'insuffisance des ressources sur la mortalité de ces patients étaient limitées.

En outre, notre modèle ne tient pas compte de la propagation de la COVID-19 dans l'hôpital, ce qui pourrait potentiellement nous amener à sous-estimer les besoins en matière de ressources. Nous n'avons pas non plus considéré de façon explicite les limites relatives aux ressources humaines et à la disponibilité de l'équipement de protection individuelle, supposant que tous les hôpitaux avaient suffisamment de personnel et de fournitures.

Nous avons également considéré la progression de l'épidémie et les ressources pour l'ensemble de l'Ontario, sans tenir compte des différences régionales dans la province.

Enfin, nous reconnaissons que le décès des patients en établissement de soins de longue durée ontariens est préoccupant. Or, comme notre modèle était axé sur les soins hospitaliers, il prenait en considération les patients des établissements de soins de longue durée qui ont été transférés à l'hôpital, mais pas ceux qui sont décédés sans avoir été transférés.

\section{Conclusion}

Grâce aux mesures de santé publique adoptées et à l'augmentation de la capacité du système de santé, les ressources hospitalières de l'Ontario semblent être suffisantes pour permettre la prise en charge des patients atteints de la COVID-19. Le scénario hypothétique dans lequel ces mesures n'auraient pas été prises donne à réfléchir, montrant l'importance de maintenir à court terme les interventions de santé publique énergiques déployées. Leur relâchement prématuré pourrait entraîner une résurgence de la COVID-19, qui aurait le potentiel de surcharger les ressources hospitalières de l'Ontario.

\section{Références}

1. Naming the coronavirus disease (COVID-19) and the virus that causes it. Genève : Organisation mondiale de la Santé. Accessible ici : www.who.int/emergencies/ diseases/novel-coronavirus-2019/technical-guidance/naming-the-coronavirus -disease-(covid-2019)-and-the-virus-that-causes-it (consulté le 20 mars 2020).

2. WHO Director-General's opening remarks at the media briefing on COVID-19March 11 2020. Genève : Organisation mondiale de la Santé.; 2020. Accessible ici : www.who.int/dg/speeches/detail/who-director-general-s-opening-remarks-at -the-media-briefing-on-covid-19---11-march-2020 (consulté le 20 mars 2020).

3. COVID-19 situation update worldwide, as of 13 April 2020. Solna (Suède) : European Centre for Disease Prevention and Control; 2020. Accessible ici : www.ecdc.europa. eu/en/geographical-distribution-2019-ncov-cases? (consulté le 13 avril 2020). 
4. Coronavirus disease (COVID-2019) situation reports. Genève : Organisation mondiale de la Santé; 2020. Accessible ici : www.who.int/emergencies/ diseases/novel-coronavirus-2019/situation-reports (consulté le 12 avril 2020).

5. Coronavirus disease 2019 (COVID-19): Situation report - 59. Genève : Organisation mondiale de la Santé; 2020. Accessible ici : www.who.int/docs/defaultsource/coronavirus/situation-reports/20200319-sitrep-59-covid-19.pdf?sfvrsn= c3dcdef9_2 (consulté le 20 mars 2020).

6. COVID-19 Community Mobility Reports. Google. Accessible ici : www.google. com/covid19/mobility/ (consulté le 11 avril 2020).

7. Murthy S, Gomersall CD, Fowler RA. Care for critically ill patients with COVID-19. JAMA le 11 mars 2020 [Cyberpublication avant impression]. doi: 10.1001/ jama.2020.3633.

8. Remuzzi A, Remuzzi G. COVID-19 and Italy: What next? Lancet 2020;395:1225-8.

9. Ferguson NM, Laydon D, Nedjati-Gilani G, et al. Imperial College COVID-19 Response Team. Report 9: Impact of non-pharmaceutical interventions (NPIS) to reduce COVID-19 mortality and healthcare demand. London (UK): Imperial College London; 2020.

10. Tuite AR, Fisman DN, Greer AL. Mathematical modelling of COVID-19 transmission and mitigation strategies in the population of Ontario, Canada. CMAJ 2020;192: E497-505.
11. Wu Z, McGoogan JM. Characteristics of and important lessons from the coronavirus disease 2019 (COVID-19) outbreak in China: summary of a report of 72314 cases from the Chinese Center for Disease Control and Prevention. JAMA le 24 février 2020 [Cyberpublication avant impression]. doi: 10.1001/jama.2020 .2648 .

12. Hospital beds. Organisation for Economic Co-operation and Development; 2018. Accessible ici : https://data.oecd.org/healtheqt/hospital-beds.htm (consulté le 25 mars 2020).

13. Coronavirus disease 2019 (COVID-19) daily epidemiology update. Public Health Agency of Canada; mis à jour le 3 mai 2020.

14. COVID-19 updates. Critical Care Services Ontario; 2020. Accessible ici : https:// criticalcareontario.com/covid-19-updates (consulté le 14 avril 2020).

15. Bellani G, Laffey JG, Pham T, et al.; LUNG SAFE Investigators; ESICM Trials Group. Epidemiology, patterns of care, and mortality for patients with acute respiratory distress syndrome in intensive care units in 50 countries. JAMA 2016;315:788-800.

16. Shoukat A, Wells CR, Langley JM, et al. Projecting demand for critical care beds during COVID-19 outbreaks in Canada. CMAJ 2020;192:E489-96.
Intérêts concurrents : Kali Barrett a reçu des honoraires personnels de Xenios AG pour des travaux autres que la présente étude. Aucun autre intérêt concurrent n'a été déclaré.

Cet article a été révisé par des pairs.

Affiliations : Institut des politiques, de la gestion et de l'évaluation de la santé (K. Barrett, Y. Khan, S. Mac, D. Naimark, B. Sander), Université de Toronto; Réseau universitaire de santé (K. Barrett, Y. Khan, R. Ximenes); Toronto Health Economics and Technology Assessment (THETA) collaborative (S. Mac, R. Ximenes, B. Sander), Réseau universitaire de santé; Hôpital Sunnybrook (D. Naimark), Toronto, Ont.

Collaborateurs : Kali Barrett, Yasin Khan, Stephen Mac et Raphael Ximenes ont contribué en parts égales et sont les auteurs principaux du manuscrit. David Naimark et Beate Sander ont contribué en parts égales et sont les auteurs en chef. Stephen Mac, Raphael Ximenes et David Naimark ont construit le modèle. Kali Barrett, Yasin Khan et
Beate Sander ont recueilli les données. Tous les auteurs ont conçu le modèle, rédigé le manuscrit original et révisé de façon critique le contenu intellectuel important; ils ont donné leur approbation finale pour la version destinée à être publiée et assument l'entière responsabilité de tous les aspects du travail.

Financement : Cette étude a été financée en partie par la Chaire de recherche du Canada sur les aspects économiques des maladies infectieuses, détenue par Beate Sander (CRC-950-232429).

Transmission des données : Les données de l'étude tirées de la littérature et de sources publiques sont accessibles aux autres chercheurs. Les données tirées de registres gouvernementaux peuvent être obtenues en faisant une demande aux agences concernées.

Accepté : 4 mai 2020

Correspondance : Beate Sander, beate.sander@theta.utoronto.ca 